\title{
Original Research \\ Novel approaches to screening for noncommunicable diseases: Lessons from Neno, Malawi
}

\author{
Chiyembekezo Kachimanga ${ }^{1}$, Katie Cundale ${ }^{1}$, Emily Wroe ${ }^{1}$, Lawrence Nazimera ${ }^{2}$, Arnold Jumbe ${ }^{2}$, \\ Elizabeth Dunbar ${ }^{1}$, Noel Kalanga ${ }^{3}$
}

1. Partners in Health/Abwenzi Pa Za Umoyo, Neno, Malawi

2. Neno District Health Office, Neno, Malawi

3. College of Medicine, University of Malawi, Blantyre, Malawi

Correspondence: Dr Chiyembekezo Kachimanga (chembekachimanga@yahoo.co.uk)

\begin{abstract}
Background
Abstract

As Malawi continues to suffer from a large burden of noncommunicable diseases (NCDs), models for NCD screening need to be developed that do not overload a health system that is already heavily burdened by communicable diseases.

Methods

This descriptive study examined 3 screening programmes for NCDs in Neno, Malawi, that were implemented from June 2015 to December 2016. The NCD screening models were integrated into existing platforms, utilising regular mass screening events in the community, patients awaiting to be seen in a combined NCD and HIV clinic, and patients awaiting treatment at outpatient departments (OPDs). Focusing on hypertension and diabetes, we screened all adults 30 years and above for hypertension using a single blood pressure cut-off of $160 / 110 \mathrm{mmHg}$, as well as adults 40 years and above for diabetes, measuring either random blood sugar (RBS) or fasting blood sugar (FBS), with referral criteria of FBS $>126 \mathrm{mg} / \mathrm{dL}$ and RBS $>200 \mathrm{mg} / \mathrm{dL}$. Data were collected on specifically designed screening registers, then entered and analysed in Excel.
\end{abstract}

Results

Over 14,000 adults ( $\geq 12$ years old) were screened for an array of common conditions at community screening events. Of these adults, $58 \%(\mathrm{n}=8133)$ and $29 \%(\mathrm{n}=4016)$ were screened for hypertension and diabetes, respectively. Nine percent $(\mathrm{n}=716)$ and $3 \%(\mathrm{n}=113)$ were referred for further hypertension and diabetes assessment respectively. At one OPD, 5818 patients (60\%) had their blood pressures measured, and among adults 30 years and above, 168 eligible adults were referred for further hypertension assessment. Since the initiation of the screening programmes, the number of patients ever enrolled for NCD care every 3 months has nearly tripled, from 40 to 114 .

Conclusions

The screening models have shown that it is not only feasible to introduce NCD screening into a public system, but screening may have also contributed to increased enrolment in NCD care in Neno, Malawi.

\section{Introduction}

Chronic noncommunicable diseases (NCDs) contribute to nearly $70 \%$ of all deaths globally. ${ }^{1}$ In 2015 , about 39.8 million deaths from chronic NCDs occurred worldwide-a $14 \%$ increase from $2005 .^{2}$ The NCD burden is projected to further increase over the next decade, with estimated NCD deaths rising to 52 million by $2030 .{ }^{1}$ Cardiovascular diseases (CVDs) contribute to nearly half of all NCD deaths worldwide. ${ }^{2}$

The major burden of chronic NCDs occurs in low- and middle-income countries, where over two-thirds of NCD deaths are reported. ${ }^{1}$ In Africa, rates of CVDs and their risk factors vary across countries, although data are still sparse due to a lack of NCD reporting systems and minimal chronic NCD research. ${ }^{3}$ However, it is known that hypertension rates vary from $6 \%$ to $48 \%$, diabetes from $0 \%$ to $16 \%$, and stroke from $0.07 \%$ to $0.3 \% .{ }^{4}$ Malawi, one of sub-Saharan Africa's poorest countries, is estimated to have prevalence rates of up to $33 \%$ and $6 \%$ for hypertension and diabetes, respectively, in adults aged 25 to 64 years. ${ }^{5} \mathrm{NCDs}$ are considered among the leading causes of death, second only to HIV/AIDS, and a recent study on inpatients in Malawi found that, while infections remain the leading cause of adult admission, NCDs were the most frequent causes of death in adults over 55 years old. ${ }^{7}$ The high burden of NCDs in Africa presents challenges to providing NCD care, as these countries also have a huge burden of communicable diseases, notably HIV/AIDS and its complications.
Despite the devastating burden of infectious diseases in Malawi, NCD care was included in Health Sector Strategic Planning (HSSP) for 2011-2016, which was closely followed by the National NCD Action Plan (NAP) for 2012-2016. ${ }^{8}$ Through these crucial documents, the government of Malawi committed to providing essential care for common NCDsincluding CVDs, cancers, epilepsy, mental health, and injuries and trauma. The government of Malawi proposed to provide the entire spectrum of care along the NCD care pathway: primary prevention of risk factors, screening, treatment and follow-up, and monitoring and evaluation for common chronic NCDs. ${ }^{6,9}$

Screening for NCDs, especially CVDs, provides the first entry to NCD diagnosis and care. Despite screening being recognised as the entry to care, routine NCD screening at community and health facilities has, historically, been deemed not feasible in Africa due to a lack of capacity within health systems. ${ }^{10}$ As screening programmes are rolled out in other settings and for other conditions in Africa, NCD screening integration with existing screening services has been advocated. ${ }^{10}$ However, limited data exist in Malawi on the feasibility of screening programmes for common NCDs, especially CVDs.

We present 3 screening models that have been used successfully as part of clinical care in Neno, Malawi, following a trend of integrated care at the service delivery level. ${ }^{11}$ We describe the screening models, results of the 


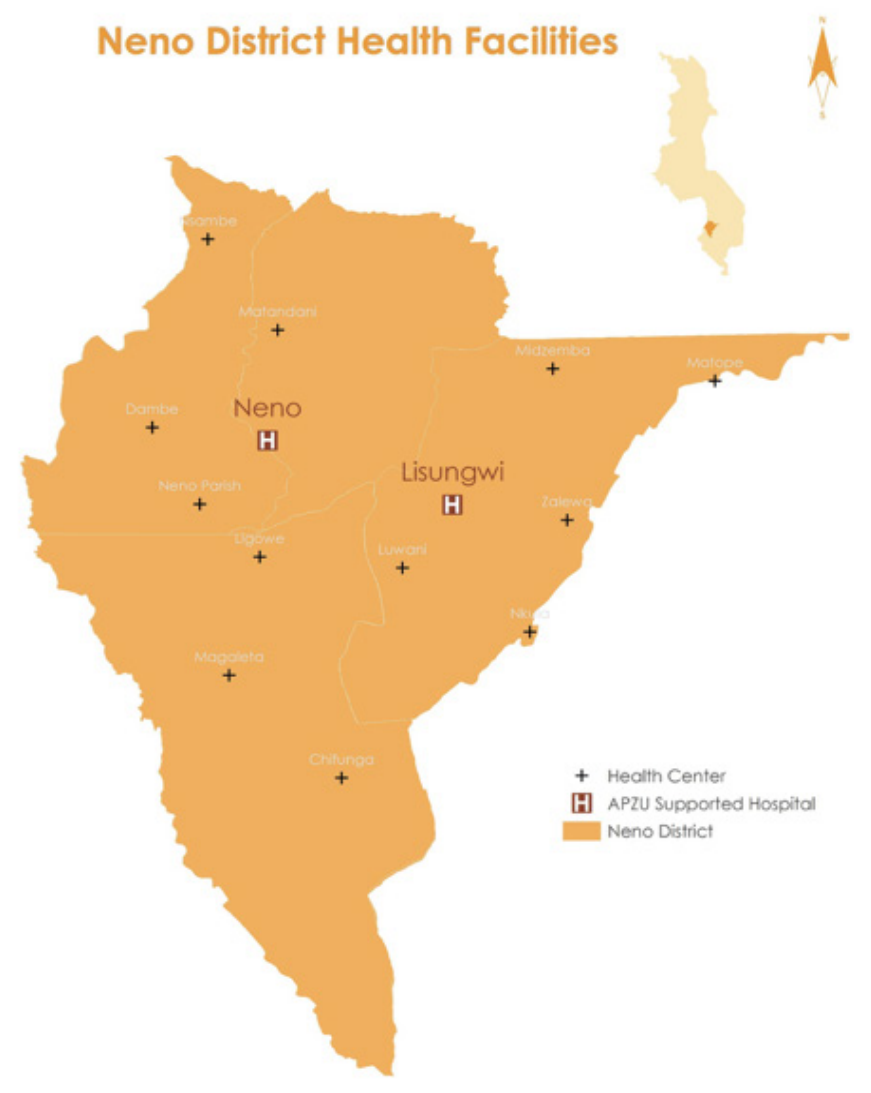

Figure 1: Map of Neno District, Malawi, showing locations of health facilities

screening programmes to date, and introduce lessons learnt from initiating these models in Neno district. We focus on multi-disease screening programmes that target nutritional disorders, hypertension, diabetes, HIV, tuberculosis (TB), and cervical cancer, but for the purpose of this descriptive paper we present data on hypertension and diabetes patients only. We hope that this adds to the growing evidence base on how to launch screening programmes for NCDs in rural areas of Malawi.

\section{Methods}

\section{Study type}

The was a descriptive study describing NCD screening programmes in the community and health facilities of Neno District, Malawi, between June 2015 and December 2016.

\section{Setting}

Partners In Health (PIH) has been working with the Ministry of Health (MOH) in Neno since 2007. Neno District is in the Southern Region of Malawi and had a estimated population of over 158,000 in 2016. ${ }^{12}$ The district has 2 hospitals and 12 health facilities (Figure 1). Access to essential care remains a challenge due to poverty and poor accessibility of health services. In Neno, about $65 \%$ of people live below the poverty line $(15 \%$ higher than national average), and about $23 \%$ of the population have limited access to health facilities. ${ }^{13,14}$ By implementing these models of multi-disease screening, we hope to expand access to care within Neno, and show that integrating NCD care into communicable disease programmes can help increase and improve service delivery without introducing considerable strain on the existing health system.

In 2009, PIH and the MOH began the Neno Chronic Care Clinic based at Neno District Hospital. This clinic was further established at Lisungwi Community Hospital in 2013. These clinics treated common NCDs, such as hypertension, asthma, epilepsy, diabetes, and congestive heart failure. ${ }^{13}$ However, in an effort to decentralise NCD care even further, the Chronic Care Clinics were integrated with HIV clinics to form the Integrated Chronic Care Clinic-locally known as $\mathrm{IC}^{3}$, or "Ice-Cubed"-in January 2015. The purpose of integration was to allow for decentralisation of NCD care to all health facilities, thus leveraging the strong platform of HIV care whilst allowing patients with NCD and HIV dual diagnoses to receive care in single combined visits. Before integration, HIV care was fully decentralised in all health facilities and over $6800 \mathrm{HIV}$ patients were receiving care in Neno as of December 2014. After the formation of $\mathrm{IC}^{3}$ in 2015, NCD care was also decentralised to all health facilities. Currently, patients with HIV, epilepsy, heart failure, hypertension, diabetes, and mental illness are all managed at $\mathrm{IC}^{3}$ at the 2 hospitals and 12 health centres in the district. Wroe et al. previously described the implementation of $\mathrm{IC}^{3}$ in Neno. ${ }^{11}$ Despite a functioning $\mathrm{IC}^{3}$ clinic, we had persistent underenrolment in NCD care in a system with limited resources available to screen for NCDs.

Before the initiation of $\mathrm{IC}^{3}$ and the screening programmes implemented shortly thereafter, very few patients in Neno were enrolled in care in comparison to the expected number of NCD patients. For example, if we accept the national prevalence estimates of $33 \%$ and $6 \%$ for hypertension and diabetes from the 2009 STEPS survey, then it would be expected that the clinics in Neno should have enrolments of close to 24,000 and 4,500 individuals with hypertension and diabetes, respectively. However, in December 2014, only 501 (representing $0.33 \%$ of Neno's population) and 66 (representing $0.044 \%$ ) had ever been enrolled in the 2 hospital-based chronic care clinics with primary diagnoses of hypertension or diabetes, respectively.

While we understood that NCDs were under-represented among patients enrolled in longitudinal care in Neno District, we did not have the resources to implement parallel NCD programmes to our pre-existing services. Therefore, while our goal was to increase case detection and enrolment in NCD care, it was to do so within the constraints of the existing health system and with limited resources.

\section{Neno NCD screening programmes}

We designed 3 screening programmes aimed at increasing case detection for NCDs: in the community, at the facility for acute outpatient care, and at $\mathrm{IC}^{3}$ itself (Table 1). The SHARC (Screening for Health and Referral in the Community) programme started as a community pilot in 2 catchment areas in February 2015, and then was scaled across the district in July 2015. During these community events, support staff screened all eligible individuals who wanted to be screened for one or more conditions, including HIV, TB, diabetes, hypertension, and nutritional disorders. A clinician was responsible for the final decision of whether or not to refer the patient to his or her nearest health facility. The facility counterpart, SHARF (Screening for Health and Referral at the Facility), offered all patients presenting to an outpatient department (OPD) the opportunity to be screened for the same set of conditions, as well as cervical cancer. Despite vital sign measurements, such as blood pressure, being a norm in developed countries and many private clinics in Malawi, most public facilities do not routinely measure vital signs. ${ }^{10}$ Therefore, we utilised patient waiting time in the OPD to 
provide targeted screening. In both these programmes, all individuals with positive screening result for NCDs were referred to $\mathrm{IC}^{3}$. At $\mathrm{IC}^{3}$ itself, we utilised patient waiting time to provide screening for enrolled patients. We trained existing $\mathrm{IC}^{3}$ clerks and expert clients to assess eligible patients and perform anthropometric measurements, blood sugar, and blood pressure. All patients who did not know their HIV status were referred to a counsellor for an HIV test, and all suspected TB patients were requested to submit sputum. Abnormal results prompted referral to an $\mathrm{IC}^{3}$ clinician for a review of these results at the same time that they were seen for their primary diagnosis. This additional NCD screening was all completed within the existing structure and workflow of $\mathrm{IC}^{3}$, allowing for additional screening without adding any extra space or staff.

These 3 screening programmes were founded on 4 basic principles. First, NCD screening should mirror current screening practices - we developed screening programmes for NCDs based on existing screening platforms in Neno, such as HIV and nutrition. Second, NCD screening should be integrated with other disease screening. This allowed the use of resources - such as vehicles, fuel, and staff timedirected to these programmes to be used for NCD screening as well. Third, task shifting should be introduced to allow for efficiency and reduce staffing costs. All disease screening is done by support staff with only 1 or 2 clinicians or nurses supporting the team. Task shifting enabled multi-disease screening to be feasible, as the district healthcare workers were too few to support all the activities of the screening programmes. Finally, all eligible individuals should be offered screening. By offering screening for more diseases to all patients, we hoped to ensure that more patients were brought into care and commenced on treatment where necessary.

\section{Screening protocols and guidelines across screening programmes}

We developed standardised guidelines for identifying eligible patients, conducting screening, capturing data, and referring patients in the 3 screening programmes. For hypertension, we screened all adults aged 30 years and older using a cut-off of $170 / 110 \mathrm{mmHg}$, in order to not overwhelm the newly decentralised $\mathrm{IC}^{3}$ clinics and to identify the highest risk patients. We then reduced this threshold to $160 / 110 \mathrm{mmHg}$ to capture more hypertensive patients. For clients with blood pressures between 140 and $159 \mathrm{mmHg}$ (systolic), and 90 and $109 \mathrm{mmHg}$ (diastolic), we provided education and encouraged a blood pressure re-check after 1 week at their nearest facility. For diabetes, we measured adults 40 years and older for either random blood sugar (RBS) if they had eaten in the previous 8 hours or fasting blood sugar (FBS) if they had not eaten in the previous 8 hours. Referral criteria for diabetic patients was FBS $>126 \mathrm{mg} / \mathrm{dL}$ and RBS > 200mg/ dL. We excluded patients who were known hypertensive or diabetic or already enrolled in our $\mathrm{IC}^{3}$ clinics with diagnoses of hypertension or diabetes. We standardised hypertension and diabetes protocols across the 3 screening programmes.

For referral, all clients with a positive screening for high blood pressure or abnormal sugar at SHARF and SHARC were given a referral slip and referred to the nearest health facility on an $\mathrm{IC}^{3}$ clinic day. At the clinic, 2 or more confirmatory blood pressure tests and fasting blood glucose tests were expected to be done and, if a clinician confirmed the diagnosis, the patient was enrolled in NCD care. If screening was done at $\mathrm{IC}^{3}$, they were seen on the same day by clinical staff.

We provided a training orientation of the protocols and guidelines to all support staff involved in the screening programmes. The training was provided to hospital attendants, expert clients, and HIV testing counsellors. Ongoing mentorship is provided by clinicians and nurses involved in all 3 screening programmes. 


\section{Data management, outcomes, and analysis}

We designed specific screening registers and forms for all 3 screening programmes. All data were recorded on paper forms, aggregated by support staff into reports, and entered into simple Microsoft Excel databases. We used Excel to conduct descriptive statistics on the clients who benefitted from the screening and were referred for further evaluation.

\section{Ethical considerations}

Since screening programmes were done within our clinical programmes and we used aggregated data in our analysis, we did not provide informed consent to the participants. We obtained approval from the Ministry of Health and National Health Sciences Research Council (protocol number 1216) to conduct the study.

\section{Results}

\section{SHARC: community-based screening}

Between July 2015 and December 2016, 178 community screening events were conducted in remote areas of Neno District, with 14,115 individuals screened for various diseases (Table 2). Of these adult SHARC clients in 2016, 58\% ( $\mathrm{n}=$ $8133)$ and $29 \%(n=4016)$ were screened for hypertension and diabetes, respectively. Among those screened, 9\% ( $\mathrm{n}=$ $716)$ and $3 \%(n=113)$ were referred to their nearest $\mathrm{IC}^{3}$ for further evaluation of hypertension and diabetes, respectively.

\section{SHARF: OPD-based screening}

SHARF was rolled out gradually, starting in May 2015. At the end of the data collection period, 5 health facilities in Neno were conducting SHARF at the OPD. One of these was Dambe Health Centre, a primary health centre where SHARF started immediately after the clinic opened in April 2016. Within the first 6 months of the health centre opening, 9691 patients visited the OPD and, of these, blood pressure was taken as a vital sign for $5818(60 \%)$ of all comers of all ages to the clinics. Among patients eligible for hypertension screening (aged $\geq 30$ years), 168 were referred to $\mathrm{IC}^{3}$ for further assessment. Diabetes screening at SHARF had not yet begun during the study period.

\section{IC': Multi-disease screening at existing HIVINCD clinics}

In June 2015, multi-disease screening at IC $^{3}$ commenced in 12 health facilities in Neno (Figure 2). Across 8 facilities between August 2015 and November 2016, 17,603 screenings took place during routine patient visits, including 11,945 and 839 screenings for hypertension and diabetes. During

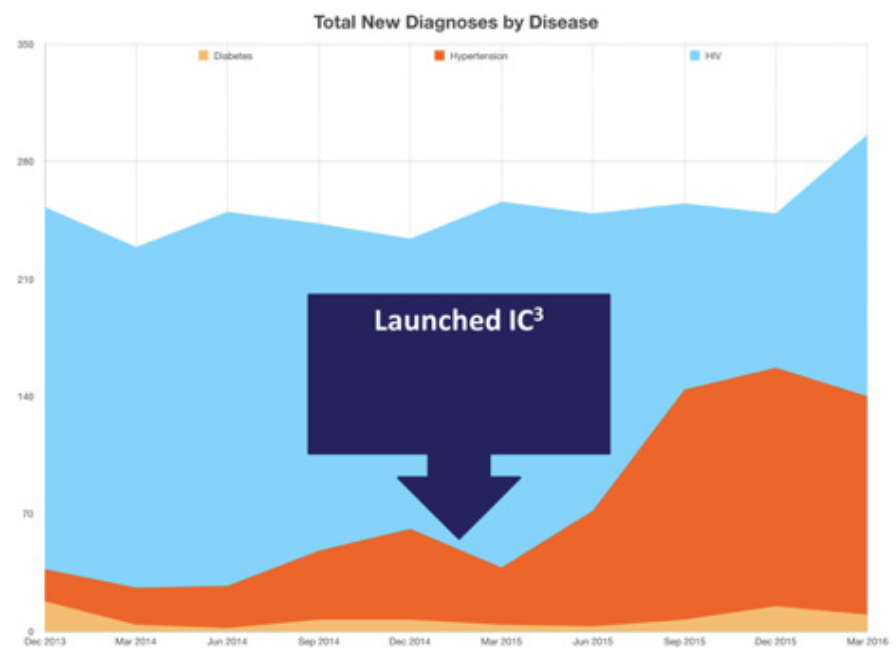

Figure 2: Increase in case detection: Launch of $\mathrm{IC}^{3}$ and initiation of noncommunicable disease screening at $\mathrm{IC}^{3}$ in June 2015
Table 2: Results from integrated community screening (SHARC) events from July 2015 to December 2016 in Neno, Malawi

$$
\text { n (\%) }
$$

\begin{tabular}{l}
$\begin{array}{l}\text { Total adults }(\geq 12 \\
\text { years old) screened }\end{array} \quad 14,150$ \\
\hline
\end{tabular}

\section{Known gender recorded}

Male $4915(34.8 \%)$

Female $8652(61.3 \%)$

Female pregnant $548(3.9 \%)$

\section{Diabetes}

Screened $4016(28.5 \%)^{*}$
Referred $113(2.8 \%)$

Hypertension

Screened $8133(57.6 \%)^{*}$

Referred $716(8.8 \%)$

SHARC $=$ Screening for Health and Referral in the

Community

*Proportion uses the number of total adults $(\geq 12$

years old) screened as the denominator, $\mathrm{N}=14,150$

this period, 2311 and 12 same-day referrals were made for hypertension and diabetes, respectively.

\section{Enrolment in IC for hypertension and diabetes}

Since the initiation of the 3 screening programmes, we saw an increase in the number of patients with NCDs enrolled in care through IC IC $^{3}$ Between October 2013 and March 2015, before start of our integrated screening programmes, we enrolled a mean of 40 new hypertensive patients per 3 -month quarter. After implementing these integrated screening programmes between April 2015 and March 2016, we enrolled an mean of 128 new hypertension clients per 3-month quarter (Figure 2).

\section{Discussion}

Our experience suggests that integrating hypertension and diabetes screening into existing community and clinic screening activities is feasible and can increase enrolment numbers of patients in longitudinal care. We employed an approach that leverages existing HIV and nutrition activities, existing staff and other resources, and employs task-shifting to carry out these pilot screening programmes. This approach has also utilised a phased screening approach by initially focusing on finding the sickest clients (stage III hypertension) in order to avoid overwhelming existing clinics and systems with new patients.

While we increased screening for NCDs and enrolment into NCD care, we recognise that much screening and enrolment remains to hit the expected number of hypertensive and diabetic individuals in Neno District. We therefore plan to expand our screening models to more communities and health facilities. By introducing SHARC events in the most rural and hard-to-reach communities, we screen patients for multiple diseases who may not travel to health facilities. 
By ensuring that SHARF is introduced to all OPD facilities in Neno, we will drastically increase the amount of people being routinely screened for NCDs and other conditions. We anticipate that by ensuring these screening programmes reach more people, our case detection and enrolment into care will also increase.

As inadequate staff, infrastructure, and training have been reported as challenges to NCD care in Malawi, we anticipated the same challenges with the $\mathrm{IC}^{3}$ clinic once the screening programmes commenced. ${ }^{8}$ Initially, we recognised that it was not possible to have a screening programme without a functioning NCD clinic, so we started the integration of the NCD screening programmes into pre-existing NCD clinics. We had a dedicated and trained team of nurses, clinicians, and support staff responsible for running all NCD clinics in Neno. As space for running the clinics is a challenge in some health facilities, especially in comparison to the number of patients attending the clinics, health centre staff continued to find solutions around NCD infrastructure at the health facility level, and we did not havec clinic cancellations due to lack of space. With the help of PIH, there was a good system of drugs and equipment forecasting, procurement, and distribution. We adjusted our equipment and drug needs to match the expected patient load and we have minimised drug stockouts for NCD care in Neno.

There are many lessons learnt from our experience in Neno District. One key limitation is the difficulty of measuring linkage to care from the point of screening into $\mathrm{IC}^{3}$. This would allow us to see whether clients referred at screening points seek follow-up care and are enrolled in care. Another limitation is our $\mathrm{IC}^{3}$ screening data, since it was captured at the visit level, not patient level. Since patients are screened at every visit and potentially make several visits a year, they can be counted more than once. It is also possible that the $\mathrm{IC}^{3}$ clerks may have screened known hypertension or diabetes patients and improperly recorded routine patient care measures as screening measures. A third limitation comes from initial use of the screening register at OPD for SHARF. Since clerks were told to take vital signs, including blood pressure, for all clients able to fit the electronic BP cuff, we were not easily able to measure proportion referred. Finally, we did not refer to $\mathrm{IC}^{3}$ some potential clients with hypertension because of the high blood pressure cut-off point for referral. To address the first limitations, we plan to launch an electronic linkage to care system that utilises a tablet-based application and bar code registration at screening events in 2017. Additionally, we plan to capture patient-level screening data at $\mathrm{IC}^{3}$ to improve screening quality and data use. We worked with clerks to write vital signs in health passport books but only recorded blood pressures in the screening register if clients were eligible for hypertension screening. We also launched a standardised monthly reporting process and form. As the clinics matures and we have better capacity to handle more patients, we will be lowering the blood pressure cutoff point gradually.

While the data collected by screening programme staff are reviewed monthly by data officers, we have learnt that a high level of oversight is needed at field events to ensure all data are collected at each screening event, and that there is a robust feedback loop to support staff at these clinics. The team continues to innovate and find new solutions around data collection systems and their application. Next steps will include further analyses to fully evaluate the costs and benefits of these integrated screening and service delivery programmes, as well as further use of data to refine screening processes and criteria.

\section{Conclusions}

To our knowledge, we are the first district in Malawi to document integrated screening programmes at the community- and facility-levels for NCDs. This has been undertaken within the public health system as part of routine clinical care. Since we did not have dedicated funding for NCD screening programmes, we leveraged resources from existing programmes to integrate further service delivery. The bulk of the work continues to be done by trained support staff, who perform the screening programmes alongside their routine duties. In an effort to continue enrolling eligible patients, we are continuing to scale up these programmes. Our experience has shown that is feasible to conduct NCD screening in a rural area of Malawi.

\section{Acknowledgements}

The authors would like to acknowledge the contributions made by Samson Njolomole and the Community Programmes team for conducting the SHARC events tirelessly since 2015, as well as the entire $\mathrm{IC}^{3}$ and SHARF teams. In addition, we wish to acknowledge the continuous hard work of the both the Ministry of Health and Partners in Health staff who tirelessly work to provide exceptional patient care for NCDs and other diseases in Neno.

\section{Competing interests}

All authors declare that they have no competing interests related to this work.

\section{References}

1. World Health Organisation. GLOBAL STATUS REPORT on non-communicable diseases. 2014.

2. Wang H, Naghavi M, Allen C, Barber RM, Bhutta ZA, Carter A, et al. Global, regional, and national life expectancy, all-cause mortality, and cause-specific mortality for 249 causes of death, 1980-2015: a systematic analysis for the Global Burden of Disease Study 2015. Lancet. 2016;388(10053):1459-544.

3. Heneghan C, Blacklock C, Perera R, Davis R, Banerjee A, Gill P, et al. Evidence for non-communicable diseases : analysis of Cochrane reviews and randomised trials by World Bank classification. BMJopen.2013: 3 :e003298. doi:10.1136/ bmjopen-2013- 003298

4. Dalal S, Beunza JJ, Volmink J, Adebamowo C, Bajunirwe F, Njelekela M, et al. Non-communicable diseases in sub-Saharan Africa : what we know now. Int. J Epidemio.2011;40:885-901.

5. Msyamboza KP, Ngwira B, Dzowela T, Mvula C, Kathyola $\mathrm{D}$, Harries $\mathrm{AD}$, et al. The burden of selected chronic noncommunicable diseases and their risk factors in malawi: Nationwide steps survey. PLoS One. 2011;6(5):6-11.

6. Ministry of Health [Malawi]. Malawi Health Sector Strategic Plan 2011-2016 - moving towards equity and quality [Internet]. 2011. Available from: http://www.medcol.mw/ commhealth/publications/3 Malawi HSSP Final Document (3).pdf

7. Allain TJ, Aston S, Mapurisa G, Ganiza TN, Banda NP, Sakala S, et al. Age Related Patterns of Disease and Mortality in Hospitalised Adults in Malawi. PLoS One [Internet]. 2017;12(1):e0168368. Available from: http://dx.plos. org/10.1371/journal.pone.0168368 
8. Lupafya PC, Mwagomba BLM, Hosig K, Maseko LM, 11. Wroe EB, Kalanga N, Mailosi B, Mwalwanda S, Chimbali H. Implementation of Policies and Strategies Kachimanga C, Nyangulu K, et al. Leveraging HIV platforms for Control of Noncommunicable Diseases in Malawi: Challenges and Opportunities. Health education and Behavior 2016;43(IS):64S-69S.

9. Ministry of Health [Malawi]. National Action plan for prevention and management of non-communicable diseases in Malawi(2012-2106). 2013.

10. Jaffar S, Amberbir A, Kayuni N, Musicha C, Nyirenda M. Viewpoint: Scaling up testing services for non-communicable diseases in Africa: Priorities for implementation research. Trop Med Int Heal. 2013;18(11):1353-6. to work toward comprehensive primary care in rural Malawi: The Integrated Chronic Care Clinic. Healthcare [Internet]. Health care.2015;3(4):270-6. Available from: http://dx.doi. org/10.1016/j.hjdsi.2015.08.002

12. Malawi National Statistical Office. 2008 Population and Housing Census: Population Projections. 2008.

13. National Statistical Office. Intergrated Household Survey (IHS 3) Report. 2012;(September):12.

14. National Statistical Office. Malawi - Demographic and Health Survey. 2011;1-603. 